\title{
Mathematical Analysis of a Series of 4-Acetylamino-2-(3,5- dimethylpyrazol-1-yl)-6-pyridylpyrimidines: A Simple Way to Relate Quantum Similarity to Local Chemical Reactivity Using the Gaussian Orbitals Localized Theory
}

\author{
Alejandro Morales-Bayuelo, ${ }^{1,2}$ Verónica Valdiris, ${ }^{3}$ and Ricardo Vivas-Reyes ${ }^{1}$ \\ ${ }^{1}$ Grupo de Química Cuántica y Teórica, Programa de Química, Facultad de Ciencias Exactas y Naturales, \\ Universidad de Cartagena, Cartagena de Indias, Colombia \\ ${ }^{2}$ Departamento de Ciencias Químicas, Universidad Nacional Andres Bello, Republica 275, 8370146 Santiago, Chile \\ ${ }^{3}$ Facultad de Medicina, Corporación Universitaria Rafael Núñez, Cartagena, Colombia
}

Correspondence should be addressed to Alejandro Morales-Bayuelo; alejandromb5@hotmail.com and Ricardo Vivas-Reyes; rvivasr@unicartagena.edu.co

Received 16 September 2013; Revised 13 January 2014; Accepted 14 January 2014; Published 16 April 2014

Academic Editor: Mihai V. Putz

Copyright (C) 2014 Alejandro Morales-Bayuelo et al. This is an open access article distributed under the Creative Commons Attribution License, which permits unrestricted use, distribution, and reproduction in any medium, provided the original work is properly cited.

\begin{abstract}
Molecular Quantum Similarity (MQS) descriptors and Density Functional Theory (DFT) based reactivity descriptors were studied for a series of 4-Acetylamino-2-(3,5-dimethylpyrazol-1-yl)-6-pyridylpyrimidines compounds used for Parkinson's disease (PD) treatment. The quantification of the steric and electronic effects was shown through scales of quantitative convergence; such scales allow us to establish a methodology to quantify the similarity from the local chemical reactivity (Fukui Functions) point of view. This procedure provides new considerations in the local reactivity of the $\mathrm{A}_{2 \mathrm{~A}}$ Adenosine receptor antagonists in a disease of difficult control as PD. In addition, we present new considerations to the localized bonding theory and show a new methodology for quantum similarity on the Fukui Functions. Considering that the Fukui functions under a condensation scheme may have ambiguities in the (DFT) context.
\end{abstract}

\section{Introduction}

Parkinson's disease (PD) is also known as idiopathic Parkinsonism or paralysis agitans [1]. PD is a chronic and degenerative disorder of the brain parts controlling the motor system. It occurs when nerve cells in the substantia nigra of the midbrain (a brain area that controls movement) die or suffer some deterioration [2]. PD is a chronic neurodegenerative disorder, which eventually leads to a progressive disability, for reasons still unknown [2-7]. PD is the second neurodegenerative disorder by their frequency, ranking behind only Alzheimer's disease [2].

PD is not only a motor system disorder. It has a wider spectrum of affectedness such as emotional wellbeing, affecting sleep, cognition, visuospatial deficits, and sensation and perception [3-5]. For all these reasons there are also other aspects of PD to be considered such as social and economic cost, for instance, within families and work places of the PD affected patients.

In this study is presented a method to quantify the steric and electronic factors in a set of 4-Acetylamino-2-(3,5dimethylpyrazol-1-yl)-6-pyridylpyrimidines as $\mathrm{A}_{2 \mathrm{~A}}$ adenosine receptor antagonists, reported by Zhang et al. [8] using the Molecular Quantum Similarity (MQS) [9-15]. Recently Bultinck and Carbó-Dorca have reported an analysis based on quantum similarity $[16,17]$, to define a link between quantum similarity and chemical reactivity. Taking into account this background, in this study we suggest a methodology for quantifying the steric and electronic effects of a series of $\mathrm{A}_{2 \mathrm{~A}}$ adenosine receptor antagonists [8]. Additionally, a series of global and local reactivity descriptors such as chemical potential $(\mu)$, hardness $(\eta)$, softness $(S)$, global electrophilic 
TABLE 1: Structures and inhibitory activity of compounds analyzed.

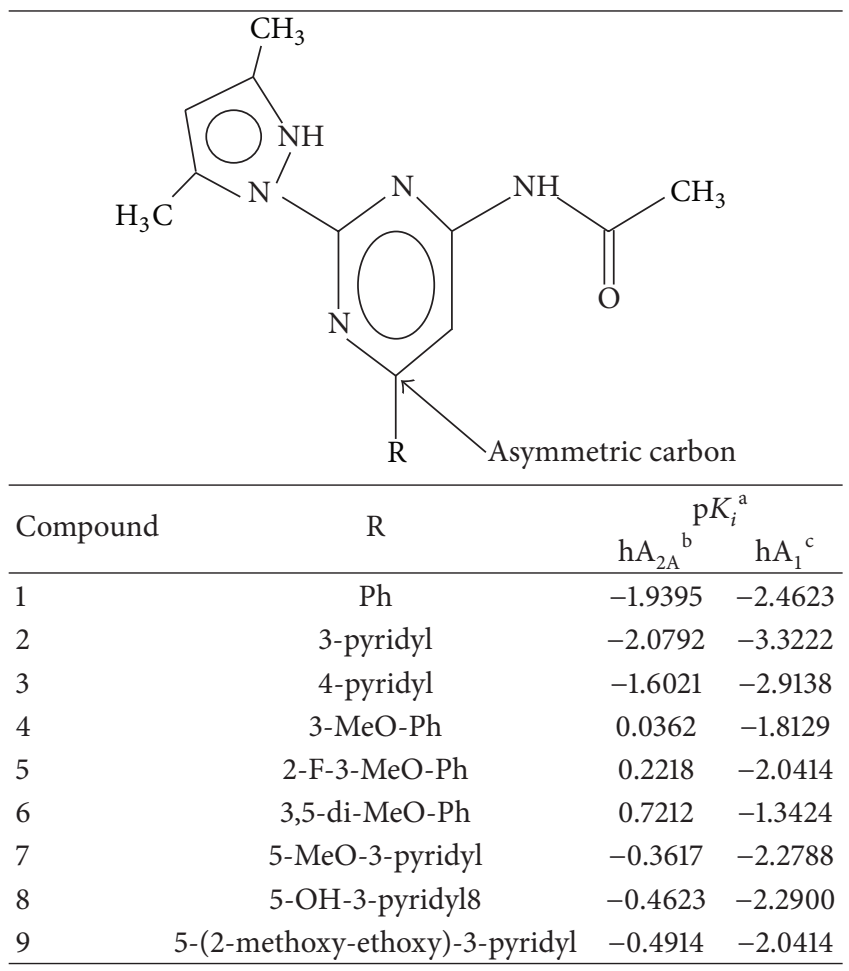

${ }^{a}$ Exp: biological activity (nM) expressed as $-\log K_{i} \mathrm{MCH}-\mathrm{R} 1$ antagonists.

${ }^{\mathrm{b}}$ Specific in the binding at $\mathrm{h} \mathrm{A}_{2 \mathrm{~A}}$ receptors expressed in HEK293 cells.

${ }^{\mathrm{c}}$ Specific in the binding at $\mathrm{h} \mathrm{A}_{1}$ receptors expressed in HEK293 cells.

$(\omega)$, and local (Fukui functions) in the Density Function Theory (DFT) context $[16,17]$ were calculated. Due to the fact that the Fukui functions can describe the local reactivity [18-20], this study presents methodologies for quantifying the similarity of the Fukui functions in the DFT context, which can be applied to a much wider range of compounds, including cases where more than one substituent group changes. Other reason, to makes this study is presents new perspectives on the topological analysis [21-23] such as the role of chemical bonding [24-26], insights into atoms in molecules [26], aromaticity [27] and presents new relations in the reactivity descriptors allowing understanding reactivity parameters as the steric and electronic factors, among others.

\section{Theory and Computational Details}

2.1. Molecular Set. A set of 4-Acetylamino-2-(3,5dimethylpyrazol-1-yl)-6-pyridylpyrimidinesreported by Zhang et al. [8] (Table 1) were studied. The adenosine plays an important role as a neuromodulator in the central nervous system through interaction with its receptors $A_{1}, A_{2 A}, A_{2 B}$, and $A_{3}$, that are widely distributed in body tissues causing vasodilation, bronchoconstriction, immune suppression, among others effects [28]. Specifically, the compounds reported by Zhang et al. are associated with receptor antagonist activity $\mathrm{A}_{2 \mathrm{~A}}[8]$.
2.2. Molecular Alignment and Computational Details. In the Molecular Quantum Similarity (MQS) field the molecular arrangement or alignment of the molecules has played a central role. Due of this dependence have been proposed by many alignment methods. Ranging from those used in CoMFA and CoMSIA methods which are in three dimensions (3D), allowing get maps steric, electronic and hydrophobic, among others [29, 30]. Gironés and CarbóDorca have implemented the alignment method based on the Topo-Geometrical Superposition Algorithm (TGSA) [31]. This method is based on the comparison of the types of atoms, distances between them, and the recognition of the largest common substructure in the aligned molecules; in this sense other alignment methods were presented in the quantum similarity [32].

In this study the similarity measures can be approximated as $\mathbf{F a}=\mathbf{F m}+\mathbf{F s i}$ and $\mathbf{F b}=\mathbf{F m}+\mathbf{F t} \mathbf{l}$ where $\mathbf{F a}$ and $\mathbf{F b}$ are density functions of two different molecules, $\mathbf{F m}$ is the common part to both functions, and Fs1, Ft1 represent the parties associated with the substituents of each compound analyzed (asymmetric carbon). The similarity provided by the partition of molecular space in this study is used to rationalize and quantify steric and electrostatic effects, through the proposition of scales of quantitative convergence, allowing us to obtain insights into atoms in molecules, aromaticity, among others $[26,27]$.

In this contribution all the molecular structures were optimized with the program Gaussian 03 [33] using DFT methods with the B3LYP exchange-correlation functional $[34,35]$, together with standard $6-31 G^{*}$ base set $[36]$.

2.2.1. Theory and Computational Details. All the geometries were optimized using the Gaussian 03 suite of programs [33] by means of Becke's three-parameter hybrid method with the Lee-Yang-Parr correlation functional, abbreviated as B3-LYP $[34,35]$, and the split valence 6-31G(d) [36].

2.2.2. Similarity Indexes. The quantum similarity field was introduced by Carbó-Dorca and coworkers [9-15]; they defined the quantum similarity measurements $Z_{A B}$ between molecules $A$ and $B$ with the electronic density $\rho_{A}(r)$ and $\rho_{B}(r)$ taking into account the minimization of the expression for the Euclidean distance as

$$
\begin{aligned}
D_{A B}^{2} & =\int\left|\rho_{A}(r)-\rho_{B}(r)\right|^{2} d r \\
& =\int \rho_{A}^{2}(r) d r+\int \rho_{B}^{2}(r) d r-2 \int \rho_{A}(r) \rho_{B}(r) d r \\
& =Z_{A A}+Z_{B B}-2 Z_{A B} .
\end{aligned}
$$

Equation (1) involves the overlap integral ZAB, often called Molecular Quantum Similarity Measure (MQSM), between the electron densities of molecules A and B. ZAA and ZBB are called the Molecular Quantum Self-Similarity Measures of 
molecules A and B [37]. Using the cosine of the angle between the density functions [15] can be expressed mathematically as

$$
I_{A B}=\frac{\int \rho_{A}(r) \rho_{B}(r) d r}{\sqrt{\int \rho_{A}(r) \rho_{A}(r) d r \int \rho_{B}(r) \rho_{B}(r) d r}}
$$

a simple manner using a general operator $(\boldsymbol{\Omega})$ can be expressed as

$$
I_{A B}=\frac{Z_{A B}(\boldsymbol{\Omega})}{\sqrt{Z_{A A}(\boldsymbol{\Omega}) Z_{B B}(\boldsymbol{\Omega})}} .
$$

The range of this index in (3) is determined by the Schwartz integral inequality mathematically defined in the interval $(0,1]$ where 1 means self-similarity and calculates only the measures of "shape similarity"; another way to obtain similarity analysis is the Hodgkin-Richards index $\operatorname{HR}\left(\mathrm{I}_{A B}\right)$ [38] that can be defined mathematically as

$$
\operatorname{HR}\left(I_{A B}\right)=\frac{2 Z_{A B}(\boldsymbol{\Omega})}{Z_{A A}(\boldsymbol{\Omega})+Z_{B B}(\boldsymbol{\Omega})} .
$$

Equation (4) is used in this study to obtain the Molecular Quantum Similarity Measurements (MQSM) and characterizations from the point of view of the atomic shells, described through the polarizability function in the B3LYP/6-31G*, that characterize the electronic population determined by steric and electronic effects in the local atomic shells; in this sense a convenient partition of the electronic density, such as the Hirshfeld Approach, is required.

\subsubsection{Local Similarity Indexes (LSI)}

(1) The Hirshfeld Approach. One of the techniques most used in quantum similarity to the partition of the electronic density was postulated by Hirshfeld [39]. This approach is based on partitioning of the electron density in a molecule by contributions $\rho(r)$. These contributions are proportional to the weight $w_{A}(r)$ of the electron density of the isolated molecule in the call promolecular density [40-42]; this weight is defined as the ratio of the electron density in the isolated atom constructed from the superposition of the density of all atoms isolated in the same position of the molecule ("the promolecular density"); this is obtained as

$$
\rho_{A}^{\text {Prom }}(r)=\sum_{x} \rho_{x}^{0}(r)
$$

To calculate the contribution of atom $A, \rho_{A}(r)$ the electron density $\rho(r)$ is obtained as

$$
\rho_{A}(r)=w_{A}(r) \rho(r) .
$$

To obtain the weight $\left(w_{A}(r)\right)$ the following equation is used:

$$
w_{A}(r)=\frac{\rho_{A}^{0}}{\sum_{x} \rho_{x}^{0}(r)},
$$

where $\rho_{A}^{0}(r)$ is the electronic density of the isolated atom $A$. Recently, using the Hirshfeld partition in quantum similarity, the global similarity index (4) at local level [43] has been calculated. In this approximation the contribution of carbon atom $\left(\mathrm{C}^{1}\right)$ in the molecule $A$ is given by

$$
\rho_{\mathrm{C}^{1}, A}(r)=w_{\mathrm{C}^{1}}(r) \rho_{A}(r)
$$

where

$$
w_{\mathrm{C}^{1}, A}=\frac{\rho_{\mathrm{C}^{1}, A}^{0}(r)}{\sum_{x} \rho_{x}^{0}(r)}
$$

equally the contribution of carbon atom $\left(C^{2}\right)$ in the molecule $B$ is obtained as

$$
\rho_{\mathrm{C}^{2}, B}(r)=w_{\mathrm{C}^{2}}(r) \rho_{B}(r)
$$

where

$$
w_{\mathrm{C}^{2}, B}=\frac{\rho_{\mathrm{C}^{2}, B}^{0}(r)}{\sum_{x} \rho_{x}^{0}(r)},
$$

so that the similarity by the product of electron density in the asymmetric carbon $\rho_{A}(r) \rho_{B}(r)$ is expressed as

$$
\rho_{\mathrm{C}, A+B}(r)=w_{\mathrm{C}, A+B}(r) \rho_{A}(r) \rho_{B}(r)
$$

getting the weighted total:

$$
w_{\mathrm{C}, A+B}(r)=\frac{\rho_{\mathrm{C}^{1}, A}^{0}(r)+\rho_{\mathrm{C}^{2}, B}^{0}(r)}{\sum_{x} \rho_{x, A}^{0}(r)+\sum_{y} \rho_{y, A}^{0}(r)},
$$

where $\sum_{x} \rho_{x, A}^{0}(r)+\sum_{y} \rho_{y, A}^{0}(r)$ is the total promolecular density of the two molecules considered, so that we can express the numerator $Z_{A B}$ Hodgkin-Richards index [38] (4) as

$$
\begin{aligned}
Z_{A, B}^{\mathrm{Local}, \mathrm{C}} & =\int w_{\mathrm{C}, A+B}(r) \rho_{A}(r) \rho_{B}(r) d r \\
& =\int\left(\frac{\rho_{\mathrm{C}^{1}, A}^{0}(r)+\rho_{\mathrm{C}^{2}, B}^{0}(r)}{\sum_{x} \rho_{x, A}^{0}(r)+\sum_{y} \rho_{y, A}^{0}(r)}\right) \rho_{A}(r) \rho_{B}(r) d r,
\end{aligned}
$$

where the global index is partitioned in its atomic contributions; this Hirshfeld approach in this study is used taking into account the holographic electron density theorem postulated by Mezey [43]. To circumvent expensive computational calculations, promolecular ASA has been used routinely to compute density functions and fitted electronic density functions from $H$ to $\mathrm{Rn}$ for use in quantum similarity measures [44].

\section{Reactivity Indexes}

In order to relate the MQS field and the local reactivity the Fukui functions are used. The Fukui function, $f(\vec{r})$, was proposed as a tool to derive the relative reactivity of different positions on a molecule by Parr and Yang [45]; also see [46]. The electron density is calculated as

$$
\rho_{A}=\sum_{v \mu} D_{v \mu} \varphi_{\nu} \varphi_{\mu}^{*},
$$


where $(D)$ represents the charge and the binding order of the matrix and $\varphi_{v}$ are the basis functions used in the iterative process of the self-consistent field (SCF). In this context the similarity measurements are obtained by the expression

$$
Z_{A B}=\sum_{\nu \in A} \sum_{\mu \in A} \sum_{\delta \in B} \sum_{\lambda \in B} D_{\nu \mu} D_{\delta \lambda} \int \varphi_{\mu}^{*}(r) \varphi_{\nu}(r) \varphi_{\lambda}^{*} \varphi_{\delta}(r) d r .
$$

To calculate the integral in (16) the classical approach of overlapping of Gaussian type orbitals is used. In this study is related the electron density with the call shape function $\sigma_{A}(r)$ according to Bultinck and Carbó-Dorca [47], this can be obtained by the relation

$$
\sigma_{A}(r)=N_{A}^{-1} \rho_{A}(r),
$$

where $N_{A}$ is the number of electrons in the molecule $A$. The shape function determines each observable of the system and may give information about the number of electrons in the electron density, despite that is Minkowski normalized to unit in all molecules:

$$
\int \sigma_{A}(r) d r=1
$$

To use this shape function we considered the relationship with several indexes as was demonstrated by Bultinck and coworkers [16, 17, 38, 48]. Using this type of mathematical considerations from the point of view of the behavior of the electronic density, we can calculate the total energy and the similarity indexes. In the chemical reactivity framework the total energy can be obtained through the use of the expression

$$
E_{v_{0}}[\rho]=F[\rho]+\int \rho(r) v_{0}(r) d r
$$

where $F$ represents the original function of Hohenberg-Kohn theorem provided by the sum of the kinetic energy $(T)$ and the functional energy electron-electron repulsion (Vee) [49, 50]. The variation of first order in the total energy is

$$
d E\left[N, v_{0}(r)\right]=\left(\frac{\partial E}{\partial N}\right)_{v_{0}(r)} d N+\int\left(\frac{\delta E}{\delta v_{0}(r)}\right)_{N} \delta v_{0}(r) d r
$$

from (19) we have

$$
\left(\frac{\delta E}{\delta v_{0}(r)}\right)_{N}=\rho(r)
$$

using (20) it is possible to define the chemical potential, through the relationship

$$
\mu \equiv\left(\frac{\partial E}{\partial N}\right)_{v_{0}(r)}
$$

Substituting (21) and (22) into (20) the following relationship is obtained:

$$
d E\left[N, v_{0}(r)\right]=\mu d N+\int \rho(r) \delta v_{0}(r) d r
$$

The chemical potential $(\mu)$ can be interpreted as the measurement of the tendency of electrons to escape from the electron cloud, whose discontinuity for integer values of $N$ was shown by Parr and Pearson [51]; the second term in (23) is called Hellmann-Feynman term [51] in the DFT context. From chemical reactivity point of view the first and second derivatives are important. The second order change in energy with respect to the number of electrons and the external potential through variations in the chemical potential is considered:

$$
d \mu\left[N, v_{0}(r)\right]=\left(\frac{\partial \mu}{\partial N}\right)_{v_{0}(r)} d N+\int\left(\frac{\delta \mu}{\delta v_{0}(r)}\right)_{N} \delta v_{0}(r) d r
$$

and electron density

$$
\begin{aligned}
\delta \rho\left[N, v_{0}(r) ; r\right]= & \left(\frac{\partial \rho(r)}{\partial N}\right)_{v_{0}(r)} d N \\
& +\int\left(\frac{\delta \rho(r)}{\delta v_{0}(r)}\right)_{N} \delta v_{0}(r) d r .
\end{aligned}
$$

In (24) the first term relates to the chemical hardness $\eta$, expressed mathematically as

$$
\eta \equiv\left(\frac{\partial \mu}{\partial N}\right)_{v_{0}(r)}=\left(\frac{\partial^{2} E}{\partial N^{2}}\right)_{v_{0}(r)} .
$$

Using (26) can quantify the opposition that puts the system to deform its electron cloud [51], while the second term in (25) represents the term called linear response function $\omega\left(r, r^{\prime}\right)$ [52]. Using the Maxwell relations in (23) is possible to define the Fukui functions [53] in (24) and (25):

$$
f(r) \equiv\left(\frac{\partial \rho(r)}{\partial N}\right)_{v_{0}(r)}=\left(\frac{\delta \mu}{\delta v_{0}(r)}\right)_{N}
$$

using (26) and (27), it is obtained for (24) and (25):

$$
\begin{gathered}
d \mu\left[N, v_{0}(r)\right]=\eta d N+\int f(r) \delta v_{0}(r) d r \\
\delta \rho\left[N, v_{0}(r) ; r\right]=f(r) d N+\int w\left(r, r^{\prime}\right) \delta v_{0}(r) d r .
\end{gathered}
$$

Interpreting the Fukui functions $(f(\vec{r}))$ from (28) and (29), we return to the question of how to explain the similarity in the Fukui functions between two molecules $A$ and $B$, using the alignment method TGSA. To answer this question we consider the case where the chemical potential of reagent $A$ is larger than the one of reagent $B$; in this case the similarity from the point of view of overlapping in the electronic distribution (16) is low, increasing the fit between the electron densities of $A$ with respect to $B$.

Now consider the case of the electronic overlap between molecules $A$ and $B$ when the electron density $\rho_{A}(r)$ tends to increase the number of electrons, $N_{A}$ :

$$
\begin{aligned}
f_{A}^{+}(r) & \equiv\left(\frac{\partial \rho_{A}(r)}{\partial N_{A}}\right)_{v_{0, A}(r)}^{+} \\
& =\lim _{\varepsilon \rightarrow 0}\left[\frac{\rho\left[N+\varepsilon, v_{0}(r)\right]-\rho\left[N, v_{0}(r)\right]}{2 \varepsilon}\right] .
\end{aligned}
$$


This implies that $f^{+}$is large in regions where there is high susceptibility to attack by nucleophilic species. Now consider the case of the electronic density $B$ versus electron density $A$ having few electrons:

$$
\begin{aligned}
f_{B}^{-}(r) & \equiv\left(\frac{\partial \rho_{B}(r)}{\partial N_{B}}\right)_{v_{0, B}(r)}^{-} \\
& =\lim _{\varepsilon \rightarrow 0}\left[\frac{\rho\left[N, v_{0}(r)\right]-\rho\left[N-\varepsilon, v_{0}(r)\right]}{2 \varepsilon}\right]
\end{aligned}
$$

from which we can conclude that the molecule $B$ donates electrons in regions where $f^{-}$is large, when the molecules $A$ and $B$ superimposed their similarity indexes in the molecular fragments depending on the similarity of chemical potential, which implies that the variations of electrons are $(\Delta \mathbf{N A} \approx$ $\Delta \mathbf{N B} \approx 0$ ) and we can obtain high overlap values in the electronic population; this electronic similarity can be related to the overlap in the Fukui functions using the concept of lateral boundaries:

$$
\begin{aligned}
f^{0}(r) & \equiv \lim _{\varepsilon \rightarrow 0} \frac{\rho\left[N+\varepsilon, v_{0}(r)\right]-\rho\left[N-\varepsilon, v_{0}(r)\right]}{2 \varepsilon} \\
& =\frac{f^{+}(r)+f^{-}(r)}{2},
\end{aligned}
$$

where we can get the following relations:

$$
I=A, \quad B: f_{I}^{0}(r) \equiv \frac{f_{I}^{+}(r)+f_{I}^{-}(r)}{2} .
$$

These expressions can be used to predict reactivity sites in neutral species.

\section{Results and Discussions}

In order to postulate a possible form to relate the MQS and local reactivity a MQS Indexes analysis, a global and local reactivity study and finally a mathematic analysis among the Fukui functions and Quantum similarity is developed.

4.1. Molecular Quantum Similarity Indexes Analysis. In Tables 3, 4, 5, and 6 the similarity matrixes used to quantify the steric and electronic effects on the different compounds studied are depicted. The similarity indexes are calculated using (1) for the Euclidean distances and (14) for the local Hodgkin-Richards index. This approach gives us information about the quantum similarity using shape function in the electron density of Gaussian type orbitals, taking into account the good correlation between the shape functions with the Hodgkin-Richards indexes demonstrated by Bultinck et al. [16, 17]. Such analysis was developed on the series of 4-Acetylamino-2-(3,5-dimethylpyrazol-1-yl)-6pyridylpyrimidines as $\mathrm{A}_{2 \mathrm{~A}}$ Adenosine receptor antagonists for the PD treatment reported by Zhang and coworkers [8].

The compounds 1 and 2 have the highest values of similarity with a value of 0.9904 (Table 2), whereas the comparison between compounds 1 and 5 give the smaller value 0.6262 . So that effect of activation on para position in the compound 3 are more relevant that the activation of the substituent in the meta position 2, in agrement with the experimental values of the $\mathrm{p} k_{\mathrm{i}}$ (see Table 1 ). Moreover, the lowest value of Euclidean distance is obtained in the substituent of compound 5 and compound 9, presenting difficulties in the structural alignment of the substituents of asymmetric carbon, to quantify the degree of alignment from the structural point of view the overlap index is displayed in Table 2.

In Table 3 is depicted the lowest Euclidean distance between the compounds 1 and 2 with a value of 0.5976 , and presents a largest value in comparison with molecules 5 and 9 with a value of 4.1730 . To quantify the similarity of the electronic orbital populations the values of GaussianRichards Hodgkin indexes in Table 4 with the corresponding Euclidean distance are calculated (Table 5).

In Table 4 are shown the Hodgkin-Richards Coulomb indexes; the higher value is found between the compounds 1 and 2 with a value of 0.9995 , according to the similarity values of overlap shown in Tables 2 and 3. Moreover, Table 4 shows the high values of the electronic similarity in compounds that have low structural similarity as is the case of compounds 5 and 9 with the Hodgkin-Richards index of overlap 0.6417 (see Table 2) and for the Coulomb similarity 0.9322, through the alignment TGSA characterized by the Euclidean distances of Coulombs (Table 5).

In Table 5 are shown the values of the Euclidean distances computed under a Coulomb operator weight. The lower value is found between molecules 1 and 2 with a value of 1.3871, showing the higher similarity electronics reported (Table 4). The higher value in the Euclidean Distance is observed when we compared the compounds 5 and 9 with a value of 19.3302 , in agreement with the greatest structural similarity shown in Table 2 .

In order to quantify the steric and electronic similarity of the compounds, we used the compound 6 that is the most active of the series according to Table 1. Figure 1 shows the steric and electronic effects in form of similarity scales of the compound 6 with respect to the other compounds.

In Figure 1 are shown the Coulomb and overlap scales on the series of receptor antagonists 4-Acetylamino-2-(3,5dimethylpyrazol-1-yl)-pyrimidines, using the most active compound of reference 6. Overlap and Coulomb scales show the same trends along of molecular set. Showing a good correlation in the quantitative method proposed for orbital similarity, allowing describe the structural and electronic similarity from the local perspective in the asymmetric carbon substituted. To determine the asymmetry from the point of view of the local chemical reactivity is calculated the global and local reactivity indexes.

4.2. Global and Local Reactivity Analysis. The global and local reactivity indexes such as Chemical potential $(\mu)$, harness $(\eta)$, and electrophilic $(\omega)$ in units of electronvoltio $(\mathrm{eV})$ are shown in Table 6.

The compound 6 has the higher chemical potential $(\mu)$ : $-3.7105 \mathrm{eV}$, (Table 6) according to the experimental values of $\mathrm{A}_{2 \mathrm{~A}}$ antagonist activity with a $\mathrm{p} K_{i}$ : 0.7212 ; see Table 1. 
TABLE 2: Molecular Quantum Similarity matrix using the overlap operator.

\begin{tabular}{lcccccccc}
\hline $\mathrm{C}^{\mathrm{a}}$ & 1 & 2 & 3 & 4 & 5 & 6 & 7 & 8 \\
\hline 1 & 1.0000 & & & & & & & \\
2 & 0.9904 & 1.0000 & & & & & \\
3 & 0.8560 & 0.8568 & 1.0000 & & & & \\
4 & 0.8194 & 0.7941 & 0.9402 & 1.0000 & & & \\
5 & 0.6262 & 0.6291 & 0.7392 & 0.6939 & 1.0000 & & & \\
6 & 0.7880 & 0.7699 & 0.8979 & 0.9526 & 0.6582 & 1.0000 & & \\
7 & 0.8160 & 0.8267 & 0.9307 & 0.8858 & 0.6909 & 0.9340 & 1.0000 & \\
8 & 0.8299 & 0.8414 & 0.9516 & 0.9011 & 0.7029 & 0.9207 & 0.9828 & 1.0000 \\
9 & 0.7592 & 0.7683 & 0.8721 & 0.8230 & 0.6417 & 0.8812 & 0.9398 & 0.9133 \\
\hline
\end{tabular}

${ }^{\mathrm{a} C}$ : compound (Table 1).

TABLE 3: Molecular Quantum Similarity matrix using the overlap Euclidean distances.

\begin{tabular}{|c|c|c|c|c|c|c|c|c|c|}
\hline $\mathrm{C}^{\mathrm{a}}$ & 1 & 2 & 3 & 4 & 5 & 6 & 7 & 8 & 9 \\
\hline 1 & 0.0000 & & & & & & & & \\
\hline 2 & 0.5976 & 0.0000 & & & & & & & \\
\hline 3 & 2.3053 & 2.3128 & 0.0000 & & & & & & \\
\hline 4 & 2.6701 & 2.8619 & 1.5564 & 0.0000 & & & & & \\
\hline 5 & 3.9976 & 3.9991 & 3.3674 & 3.7111 & 0.0000 & & & & \\
\hline 6 & 3.0183 & 3.1491 & 2.1341 & 1.4754 & 4.0249 & 0.0000 & & & \\
\hline 7 & 2.7161 & 2.6472 & 1.6896 & 2.1961 & 3.7456 & 1.7348 & 0.0000 & & \\
\hline 8 & 2.5811 & 2.5039 & 1.3952 & 2.0253 & 3.6478 & 1.8932 & 0.8521 & 0.0000 & \\
\hline 9 & 3.2722 & 3.2207 & 2.4315 & 2.8626 & 4.1730 & 2.3955 & 1.6992 & 2.0240 & 0.0000 \\
\hline
\end{tabular}

TABLE 4: Molecular Quantum Similarity matrix using the Coulomb operator.

\begin{tabular}{ccccccccc}
\hline $\mathrm{C}^{\mathrm{a}}$ & 1 & 2 & 3 & 4 & 5 & 6 & 7 & 8 \\
\hline 1 & 1.0000 & & & & & & & \\
2 & 0.9995 & 1.0000 & & & & & & \\
3 & 0.9802 & 0.9803 & 1.0000 & & & & \\
4 & 0.9694 & 0.9668 & 0.9877 & 1.0000 & & & \\
5 & 0.9471 & 0.9461 & 0.9653 & 0.9609 & 1.0000 & & & \\
6 & 0.9582 & 0.9567 & 0.9762 & 0.9898 & 0.9517 & 1.0000 & 0.0000 & 1.0000 \\
7 & 0.9690 & 0.9690 & 0.9872 & 0.9793 & 0.9574 & 0.9889 & 0.9967 & 0.9705 \\
8 & 0.9755 & 0.9760 & 0.9946 & 0.9841 & 0.9610 & 0.9848 & 1.0000 \\
9 & 0.9387 & 0.9384 & 0.9571 & 0.9519 & 0.9322 & 0.9735 & 0.9813 &
\end{tabular}

TABLE 5: Molecular Quantum Similarity matrix using the Coulomb Euclidean distances.

\begin{tabular}{ccccccccc}
\hline $\mathrm{C}^{\mathrm{a}}$ & 1 & 2 & 3 & 4 & 5 & 6 & 7 & 8 \\
\hline 1 & 0.0000 & & & & & & & \\
2 & 1.3871 & 0.0000 & & & & & \\
3 & 9.0371 & 9.0286 & 0.0000 & & & & \\
4 & 12.1128 & 12.5541 & 8.0907 & 0.0000 & & & \\
5 & 16.6637 & 16.7852 & 13.9059 & 14.1616 & 0.0000 & & & \\
6 & 15.6116 & 15.8052 & 12.5861 & 7.9836 & 16.0839 & 0.0000 & & \\
7 & 12.1970 & 12.1811 & 8.2480 & 9.9421 & 14.7569 & 8.2327 & 0.0000 & 0.0000 \\
8 & 10.4197 & 10.3080 & 5.1554 & 8.7034 & 14.2522 & 9.9648 & 4.1009 & 10.7887 \\
9 & 18.8930 & 18.9020 & 16.3670 & 16.4250 & 19.3302 & 12.1781 & 13.5562 & 0.0000 \\
\hline
\end{tabular}

\footnotetext{
${ }^{\mathrm{a}} \mathrm{C}$ : compound (Table 1).
} 

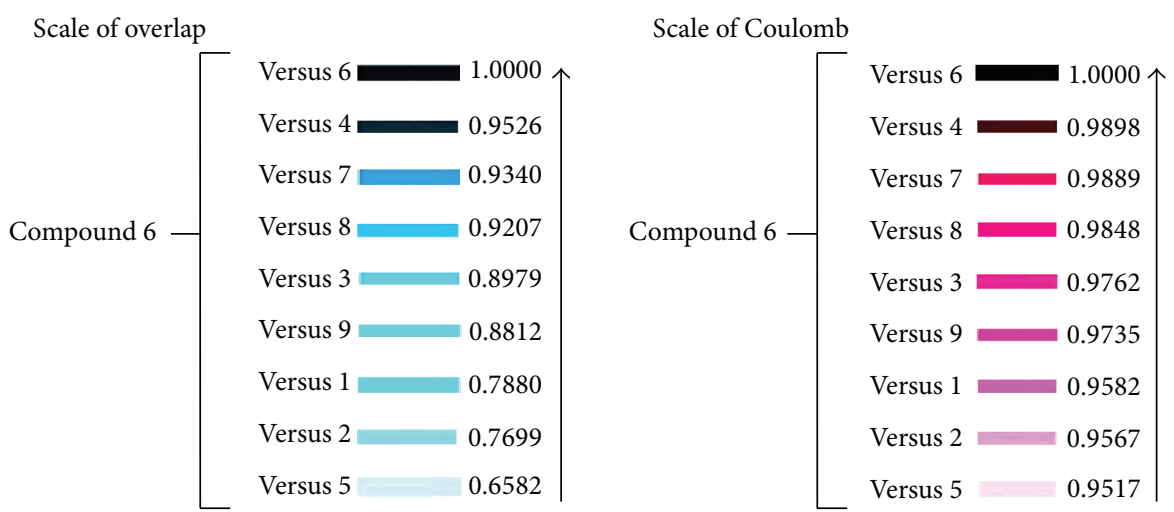

FIgURE 1: Overlap and Coulomb scales of quantitative convergence, proposed in this study, using (14), for the (MQSM).

TABLE 6: Global and local reactivity indexes.

\begin{tabular}{lccc}
\hline Compound & Chemical P $(\mu)$ & Hardness $(\eta)$ & Electrophilicity $(\omega)$ \\
\hline 1 & -3.9711 & 4.6763 & 1.6861 \\
2 & -4.1410 & 4.5661 & 1.8778 \\
3 & -4.2797 & 2.0646 & 2.0646 \\
4 & -3.8978 & 4.5940 & 1.6534 \\
5 & -3.8836 & 4.7059 & 1.6025 \\
6 & -3.7105 & 4.3745 & 1.5737 \\
7 & -4.0952 & 4.5457 & 1.8447 \\
8 & -4.1378 & 4.5138 & 1.8965 \\
9 & -4.1043 & 4.5340 & 1.8577 \\
\hline
\end{tabular}

${ }^{*}$ Unit $(\mathrm{eV})$.

Moreover, the compound more stable is the compound 3 with $\mu$ : $-4.2797 \mathrm{eV}$ and a $\mathrm{p} K_{i}$ value: -1.6021 . This Compound also has the higher hardness $\eta$ : $4.3745 \mathrm{eV}$, and allows understanding the greater tendency of the electrons to escape from the electron cloud with the substituent group 3,5-di-MeO$\mathrm{Ph}$, and the greatest opposition to distort the electron cloud in the common structural fragment in the test range. This relationship is also seen in the less reactive compound 3 .

The stabilization energy of the system when it is saturated by electrons is calculated with the electrophilic index $(\omega)$, the compound 3 is the lees reactive and it has the highest electrophilic, this is determined by the substituent group (4pyridyl); see Table 1 . These considerations are according to the reactivity and with the lower value in the hardness $\eta$ : $2.0646 \mathrm{eV}$ allowing relating the degree of distortion of the electron cloud with a low energy needed by the system when it has electronic saturation according to equation $(\omega: \mu 2 / 2 \eta)$. Allowed us relate a low reactivity with a high electrophilicity in the Gaussian orbital, describing the asymmetry of the carbon atom and of the substituents studied. In this sense, the local indexes are calculated to determine this condition and the local reactivity through the Fukui functions that are shown in Table 7.

As the substituents $\mathrm{R}(6)$ with the $\mathrm{R}(4)$ differ only by the presence of methoxy (MeO-), this (dis)similarity in the asymmetric carbon between the two structures is due to the C33 carbon atom determinate by molecular alignment TGSA and is quantified with the overlap similarity index 0.9526 , see Table 2, and with the electronic similarity 0.9898, see Table 4 .

The consideration of the local chemical reactivity of the Fukui functions was derived taking into account the lateral equations ((30) and (31)) with 0.0552 to compound 6 , and for the compound 4:0.0196, these differences in the local reactivity were quantified through the (dis)similarity in the electronic population from the point of view of the Gaussian orbitals (16) and using the shape function in the ASA approach [54].

To establish the conditions of similarity in the chemical reactivity between the two structures 6 and 4 (see Table 1), we can use the relations

$$
\begin{gathered}
f_{\mathrm{C} 33}^{0}(6)=\lim _{\mathrm{IS} \rightarrow 1}\left\{f_{\mathrm{C} 33}^{0}(4)\right\} \\
\lim _{\varepsilon \rightarrow 0} \frac{\rho_{6}^{\mathrm{C} 33}\left[N+\varepsilon, v_{0}(r)\right]-\rho_{6}^{\mathrm{C} 33}\left[N-\varepsilon, v_{0}(r)\right]}{2 \varepsilon} \\
=\lim _{\mathrm{IS} \rightarrow 1}\left\{\lim _{\varepsilon \rightarrow 0} \frac{\rho_{4}^{\mathrm{C} 33}\left[N+\varepsilon, v_{0}(r)\right]-\rho_{4}^{\mathrm{C} 33}\left[N-\varepsilon, v_{0}(r)\right]}{2 \varepsilon}\right\}, \\
\frac{f_{\mathrm{C} 33}^{+}(6)+f_{\mathrm{C} 33}^{-}(6)}{2}=\lim _{\mathrm{IS} \rightarrow 1}\left\{\frac{f_{\mathrm{C} 33}^{+}(4)+f_{\mathrm{C} 33}^{-}(4)}{2}\right\}
\end{gathered}
$$

with

$$
f_{\mathrm{C} 33}^{0}(6)=\lim _{\mathrm{ED} \rightarrow 0}\left\{f_{\mathrm{C} 33}^{0}(4)\right\},
$$

where (IS) in (34) represents the Hodgkin-Richards indexes of overlap and Coulomb, respectively. On the other hand, the self-similarity in the Fukui functions occurs when (IS $=1$ ) and in (37) the (Euclidean Distance $=\mathrm{ED}=0$ ) of Coulomb and overlap. From the point of view of the global reactivity descriptors the self-similarity occurs when

$$
\mu_{A}(6) \approx \mu_{B}(4) \quad \text { with } \Delta N_{A}(6) \approx \Delta N_{A}(4) .
$$

Equation (38) provides information about the local electronic population determined by steric considerations of the substituent groups (R), according to the methodology of alignment that is shown in Figure 2 in Table 7. Equation (38) 
TABLE 7: Fukui function, in the molecular fragment of the most reactive compound 6 with respect to the compound 4, of the substitute groups $(\mathrm{R}(n))$ in Figure 2; see Table 1.

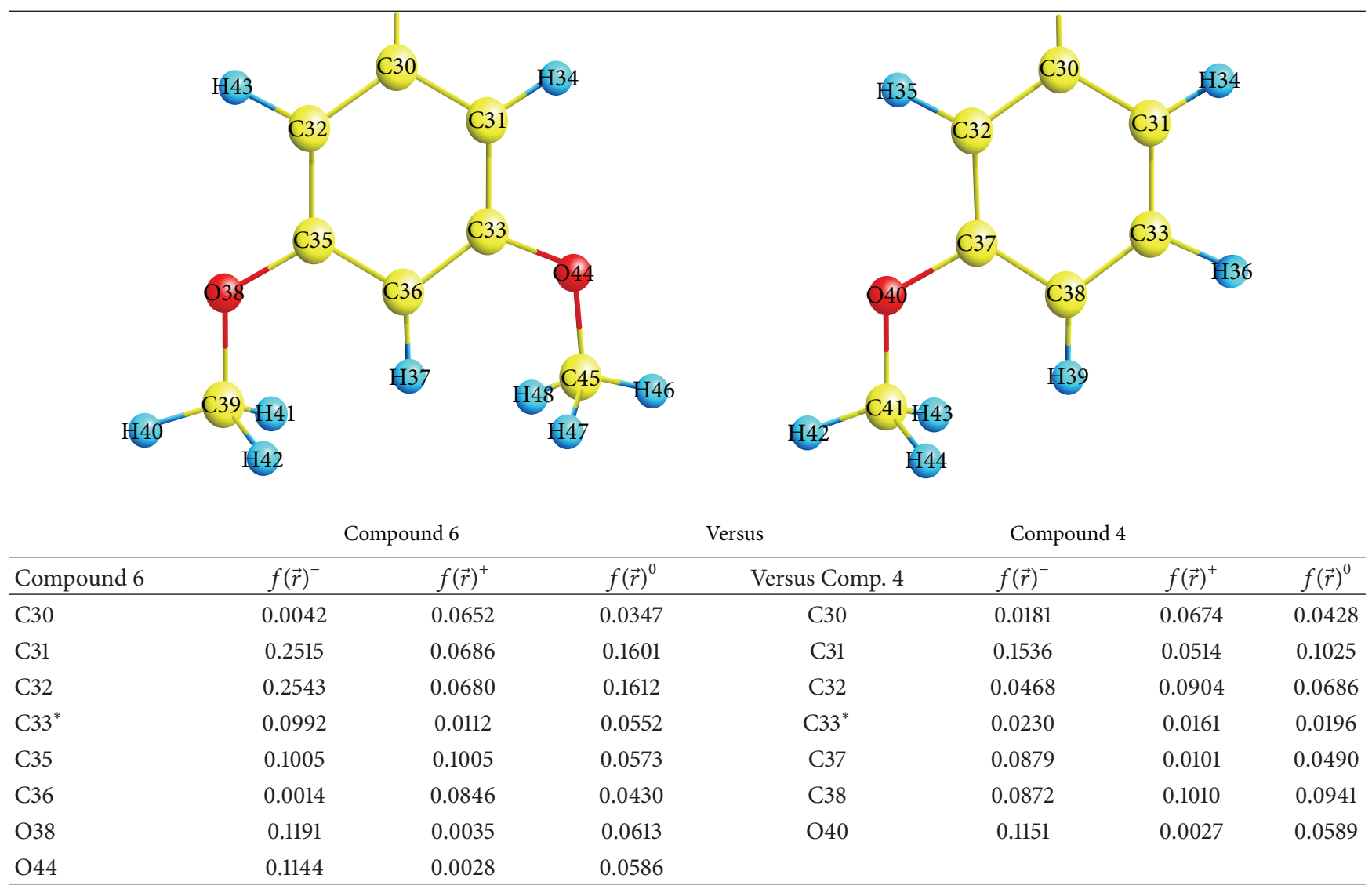

${ }^{*}$ Carbon asymmetric in the substituent $\mathrm{R}$ of compound 6 with respect to compound 4; see Table 1.

can be related con the Mulliken valence and in this sense understand the electronic population, according to Putz [55].

This measure is calculated taking the corresponding part of the squared norm of the density function belonging to the fragment of interest, so we can write

$$
Z_{A B}^{X}(\boldsymbol{\Omega})=\iint \rho_{A}^{X}\left(r_{1}\right) \boldsymbol{\Omega}\left(r_{1}, r_{2}\right) \rho_{B}^{X}\left(r_{2}\right) d r_{1} d r_{2},
$$

where $X=$ Molecular Fragment, than can quantify the asymmetric carbon (see Table 1). This measure is based on the holographic theorem of the electronic density, which ensures that the information contained in the total electron density of a molecule is also present in the local density of any molecular fragment [55].

This method provides theoretical considerations about topological similarity analysis of the Fukui functions relating it to the local similarity and presenting new topological relationships, considering that the electron localization concept in the descriptive chemical is very important; due to the fact that the Hartree-Fock canonical orbitals are delocalized over the molecular space, this method can be complementary to any analysis proposed of the molecular topology as the developed by Bader [56]. Showing new considerations to the chemical bond theory located using Fukui function; considering that the structures (see Table 1) differ by only one substituent group and each electronic density has its own distinctive topology which provides information about the nature of chemical bonding to each fragment associated with the local molecular bond considered.

In this sense, this study presents new considerations on the local reactivity through Fukui function. Considering that Fukui functions under a condensation scheme may have ambiguities according to Bultinck et al. [57]. For this reason, a mathematic analysis among the Fukui functions and quantum similarity was made to search for new links in the molecular topology, aromaticity [25], and reactivity descriptors [46].

4.3. Mathematical Analysis among the Fukui Functions and Quantum Similarity. Taking in account the recent advances of atoms in molecules showing a closely relation between DFT and electron delocalization [24], in this section we study the similarity in the aromaticity. In this section was study the similarity in the aromaticity. In order to study 41 from the mathematic point of view the relation between quantum similarity and the Fukui function, the case of the self-similarity due to the equality of the chemical potential is considered in (38):

$$
\mu_{A}=\mu_{B}=\mu_{A B}
$$


for the change of the electron population is considered the case:

$$
d N_{A}=d N_{B}=d N_{A B}
$$

In this condition the external potential also has a (dis)similarity that we can see in the chemical potential according to (38); therefore using (28) we have

$$
\begin{aligned}
d \mu_{A} & =\eta_{A} d N_{A}+\int f_{A}(r) d v_{A}(r) d r \\
& =\eta_{B} d N_{B}+\int f_{B}(r) d v_{B}(r) d r=d \mu_{B}
\end{aligned}
$$

where

$$
d v_{A}(r)=\int \frac{\delta \rho_{B}(r)}{\left|r-r^{\prime}\right|} d r^{\prime}
$$

with

$$
\delta \rho_{B}(r)=\left.\frac{\delta \rho_{B}(r)}{\delta N_{B}}\right|_{v} d N_{B}=f_{B}(r) d N_{B} .
$$

Taking into account (27) and using the same analysis of (43) to $d v_{B}$, is obtain for (42) through the Fukui functions ((33) and (35)):

$$
\begin{gathered}
\eta_{A} d N_{A}+\int f_{A}^{0}(r) d v_{A}(r) d r \\
+\left[\iint \frac{f_{A}^{0}(r) f_{B}^{0}\left(r^{\prime}\right) d r d r^{\prime}}{\left|r-r^{\prime}\right|}\right] d N_{B} \\
=\eta_{B} d N_{B}+\int f_{B}^{0}(r) d v_{B}(r) d r \\
+\left[\iint \frac{f_{A}^{0}(r) f_{B}^{0}\left(r^{\prime}\right) d r d r^{\prime}}{\left|r-r^{\prime}\right|}\right] d N_{A} .
\end{gathered}
$$

Taking into account (41), with $d v_{A}(r)=d v_{B}(r)$, we have for $d N$

$$
\begin{aligned}
{\left[\left(\eta_{A}-J\right)+\left(\eta_{B}-J\right)\right] d N=} & \int f_{B}^{0}(r) d v(r) d r \\
& -\int f_{A}^{0}(r) d v(r) d r
\end{aligned}
$$

where

$$
J=\iint \frac{f_{A}^{0}(r) f_{B}^{0}\left(r^{\prime}\right) d r d r^{\prime}}{\left|r-r^{\prime}\right|}
$$

Equation (47) is called coulomb equation and calculates the similarity determined by the quantum TGSA molecular alignment (see Table 4). Solving the equation for $d N$ in (46) is obtained:

$$
d N=\frac{\int\left[f_{B}^{0}(r)-f_{A}^{0}(r)\right] d v(r) d r}{\left(\eta_{A}+\eta_{B}\right)-2 J} .
$$

In the case of the self-similarity we have for $(48)(d N=0)$; this is due to the fact that $\mathbf{A}=\mathbf{B}$, and therefore in the coulomb integral $\mathbf{J}=\mathbf{1}$. This value for $d N=0$ can be related to the value of the Euclidean distance $D_{A B}=0$ (see Table 5) in terms of TGSA molecular alignment (see Figure 1). Thus, we can write a general equation for the Euclidean distance using (1):

$$
\begin{aligned}
D_{A B} & =\sqrt{Z_{A}+Z_{B}-2 Z_{A B}}=d N \\
& =\frac{\int\left[f_{B}^{0}(r)-f_{A}^{0}(r)\right] d v(r) d r}{\left(\eta_{A}+\eta_{B}\right)-2 J} \\
& =\frac{\int\left[f_{B}^{0}(r)-f_{A}^{0}(r)\right] d v(r) d r}{\left(\eta_{A}+\eta_{B}\right)-2\left\{\iint\left(f_{A}^{0}(r) f_{B}^{0}\left(r^{\prime}\right) d r d r^{\prime} /\left|r-r^{\prime}\right|\right)\right\}}
\end{aligned}
$$

and in terms of the chemical $\mathrm{P}(\mu)$ and electrophilicity $(\omega)$ as

$$
\begin{aligned}
D_{A B}=\int\left[f_{B}^{0}(r)-f_{A}^{0}(r)\right] d v(r) d r \\
\times\left(\left(\frac{\mu^{2}}{2 \omega}\right)_{A}+\left(\frac{\mu^{2}}{2 \omega}\right)_{B}\right. \\
\\
\left.\quad-2\left\{\iint \frac{f_{A}^{0}(r) f_{B}^{0}\left(r^{\prime}\right) d r d r^{\prime}}{\left|r-r^{\prime}\right|}\right\}\right)^{-1},
\end{aligned}
$$

where $(\eta, \mu, \omega)$ were calculated in Table 6 . Therefore, we can say that a high electronic similarity in the aromaticity between $A$ and $B$ determines a maximum molecular alignment, allowing getting the conditions of quantum similarity of the Fukui functions in terms of global reactivity descriptors and the coulomb integral. Finally, we can obtain the Carbó index, using (1) and (3):

$$
\begin{aligned}
& I_{A B}= \frac{D_{A B}^{2}-\left(Z_{A A}+Z_{B B}\right)}{-2}\left[\frac{Z_{A B}}{\sqrt{Z_{A A} Z_{B B}}}\right] \\
&=\left\{\int\left[f_{B}^{0}(r)-f_{A}^{0}(r)\right] d v(r) d r\right. \\
& \times\left(\left(\frac{\mu^{2}}{2 \omega}\right)_{A}+\left(\frac{\mu^{2}}{2 \omega}\right)_{B}\right. \\
&\left.-2\left\{\int \frac{f_{A}^{0}(r) f_{B}^{0}\left(r^{\prime}\right) d r d r^{\prime}}{\left|r-r^{\prime}\right|}\right)^{-1}\right\}^{2} \\
&\left.-\left(Z_{A A}+Z_{B B}\right)\right\}^{-1}\left[\frac{Z_{A B}}{\sqrt{Z_{A A} Z_{B B}}}\right]
\end{aligned}
$$


and for the Hodgkin-Richards index (4), used in this study:

$$
\begin{aligned}
& \operatorname{HR}\left(I_{A B}\right)=\frac{D_{A B}^{2}-\left(Z_{A A}+Z_{B B}\right)}{-2}\left[\frac{2 Z_{A B}(\boldsymbol{\Omega})}{Z_{A A}(\boldsymbol{\Omega})+Z_{B B}(\boldsymbol{\Omega})}\right] \\
& =\left(\iint\left[f_{B}^{0}(r)-f_{A}^{0}(r)\right] d v(r) d r\right. \\
& \times\left(\left(\frac{\mu^{2}}{2 \omega}\right)_{A}+\left(\frac{\mu^{2}}{2 \omega}\right)_{B}\right. \\
& \left.\left.-2\left\{\iint \frac{f_{A}^{0}(r) f_{B}^{0}\left(r^{\prime}\right) d r d r^{\prime}}{\left|r-r^{\prime}\right|}\right\}\right)^{-1}\right\}^{2} \\
& \left.-\left(Z_{A A}+Z_{B B}\right)\right) \times-2^{-1}\left[\frac{2 Z_{A B}}{Z_{A A}+Z_{B B}}\right] \text {. }
\end{aligned}
$$

Using (51) and (52) we can obtain the global quantum similarity of the Fukui functions from the electronic point of view, through the Carbó and Hodgkin-Richards index, respectively. Due to the direct proportionality among the coulomb and overlap operators is shows the similarity of the Fukui functions from point of view steric through the Dirac delta in (50) as

$$
\begin{aligned}
& D_{A B} \\
& =\frac{\int\left[f_{B}^{0}(r)-f_{A}^{0}(r)\right] d v(r) d r}{\left(\frac{\mu^{2}}{2 \omega}\right)_{A}+\left(\frac{\mu^{2}}{2 \omega}\right)_{B}-2\left\{\iint f_{A}^{0}(r) \delta\left(r-r^{\prime}\right) f_{B}^{0}\left(r^{\prime}\right) d r d r^{\prime}\right\}}
\end{aligned}
$$

obtaining for the Carbó index of (51):

$$
\begin{aligned}
I_{A B}=\left(\left\{\int\left[f_{B}^{0}(r)-f_{A}^{0}(r)\right] d v(r) d r\right.\right. \\
\quad \times\left(\left(\frac{\mu^{2}}{2 \omega}\right)_{A}+\left(\frac{\mu^{2}}{2 \omega}\right)_{B}\right. \\
\left.\left.\quad-2\left\{\iint f_{A}^{0}(r) \delta\left(r-r^{\prime}\right) f_{B}^{0}\left(r^{\prime}\right) d r d r^{\prime}\right\}\right)^{-1}\right\}^{2} \\
\left.\quad-\left(Z_{A A}+Z_{B B}\right)\right) \times-2^{-1}\left[\frac{Z_{A B}}{\sqrt{Z_{A A} Z_{B B}}}\right]
\end{aligned}
$$

and for the Hodgkin-Richards index (52)

$$
\begin{aligned}
& \operatorname{HR}\left(I_{A B}\right) \\
& =\left(\iint\left[f_{B}^{0}(r)-f_{A}^{0}(r)\right] d v(r) d r\right. \\
& \quad \times\left(\left(\frac{\mu^{2}}{2 \omega}\right)_{A}+\left(\frac{\mu^{2}}{2 \omega}\right)_{B}\right. \\
& \left.\left.\quad-2\left\{\int f_{A}^{0}(r) \delta\left(r-r^{\prime}\right) f_{B}^{0}\left(r^{\prime}\right) d r d r^{\prime}\right\}\right)^{-1}\right\}^{2} \\
& \left.\quad-\left(Z_{A A}+Z_{B B}\right)\right) \times-2^{-1}\left[\frac{2 Z_{A B}}{Z_{A A}+Z_{B B}}\right]
\end{aligned}
$$

with the interval of $(0,1]$ according to the Schwartz integral inequality [58]:

$$
\left[\int \rho_{A}(r) \rho_{B}(r) d r\right]^{2} \leq \int \rho_{A}^{2}(r) d r \int \rho_{B}^{2}(r) d r,
$$

where $\rho_{A}(r)$ and $\rho_{B}(r)$ are the electron densities considered.

In this sense, new considerations on the quantum similarity field to relate the local selectivity from the electronic ((51) and (52)) and steric ((54) and (55)) point of view are presented. On the other hand, taking into account the role of the Fukui functions [58] in the chemistry reactivity and the mathematical approximations to compute such functions $[57,59]$ is necessary presents others mathematical methods that help to characterize the chemical bonds within the topological analysis.

Moreover, the coherence among the overlap and coulomb scales of quantitative convergence reported in this study (see Figure 2 in Table 7) is very important taking in account that the structures, their properties, and reactivity parameters in the compounds studies dependent strictly on the nature of covalent bond in the asymmetric carbon (see Table 1). These bonds provide a basic skeleton of the molecule easily modifiable by the repulsive forces in the case of bulky substituents. Therefore, complements with the electrostatic effects are involved, as for example induction, aromaticity, the molecular symmetry and the electrostatic interactions calculated by the coulomb operator.

\section{Conclusions and Perspectives}

A theoretical method to quantify the steric and electronic effects in a series of 4-Acetylamino-2-(3,5-dimethylpyrazol1-yl)-6-pyridylpyrimidines as $\mathrm{A}_{2 \mathrm{~A}}$ Adenosine receptor antagonists for the PD treatment, using Quantum Molecular similarity and global and local reactivity descriptors within of DFT context, was proposed. The overlap and Coulomb Hodgkin-Richards indexes are shown in form of quantitative convergence scales, obtaining the same trend of similarity in both scales, presenting a systematization method of steric and electronic effects in this type of inhibitors that can be 
extended to a much larger series of inhibitors for the PD treatment.

To carry out the similarity in local reactivity, the TGSA alignment method to solve the open problem of optimal alignment in quantum similarity is taken. Giving, new considerations on the Gaussian orbitals localized theory from the quantum similarity and the local reactivity, which can be applied in a much broader range of receptors antagonists $\mathrm{A}_{2 \mathrm{~A}}$ and understand from the electronic and structural point of view the experimental behavior of these compounds, that may be considered in drug design for the treatment of a disease of difficult control as the PD.

Presenting new considerations and alternatives for the characterization of the Fukui functions and taking into account that the Fukui function under a condensation scheme may have ambiguities according to Fukui [58]. In this sense, this contribution presents new insight to create systematic methodologies on the MQS analysis in the Quantitative Structural Activity Relationship (QSAR) methodology.

\section{Conflict of Interests}

The authors declare that there is no conflict of interests regarding the publication of this paper.

\section{Acknowledgments}

Alejandro Morales-Bayuelo thanks the Universidad Nacional Andres Bello (Santiago, Chile) for a Ph.D. fellowship (CONICYT (63100003)). Ricardo Vivas-Reyes wishes to thank the Universidad de Cartagena (Cartagena de Indias, Colombia), for continuous support to his group. Finally, The authors thank Ramon Carbó-Dorca (Universidad de Girona, España) and Mihai V. Putz (Lab. Computational and Structural Physical-Chemistry for Nanosciences and QSAR West University of Timisoara Biology-Chemistry department, Rumania) for their important contribution.

\section{References}

[1] J. Jankovic, "Parkinson's disease: clinical features and diagnosis," Journal of Neurology, Neurosurgery \& Psychiatry, vol. 79, pp. 368-376, 2008.

[2] C. A. Davie, "A review of Parkinson's disease," British Medical Bulletin, vol. 86, no. 1, pp. 109-127, 2008.

[3] M. Barnett-Cowan, R. T. Dyde, S. H. Fox, E. Moro, W. D. Hutchison, and L. R. Harris, "Multisensory determinants of orientation perception in Parkinson's disease," Neuroscience, vol. 167, no. 4, pp. 1138-1150, 2010.

[4] D. Garcia-Borreguero, O. Larrosa, and M. Bravo, "Parkinson's disease and sleep," Sleep Medicine Reviews, vol. 7, no. 2, pp. 115129,2003

[5] M. Vaugoyeau, S. Viel, C. Assaiante, B. Amblard, and J. P. Azulay, "Impaired vertical postural control and proprioceptive integration deficits in Parkinson's disease," Neuroscience, vol. 146, no. 2, pp. 852-863, 2007.

[6] D. Aarsland, E. Londos, and C. Ballard, "Parkinson's disease dementia and dementia with Lewy bodies: different aspects of one entity," International Psychogeriatrics, vol. 21, no. 2, pp. 216219, 2009.

[7] L. M. de Lau and M. M. Breteler, “Epidemiology of Parkinson's disease," The Lancet Neurology, vol. 5, no. 6, pp. 525-535, 2006.

[8] X. Zhang, J. E. Tellew, Z. Luo et al., "Lead optimization of 4-acetylamino-2-(3,5-dimethylpyrazol-1-yl)-6pyridylpyrimidines as $\mathrm{A} 2 \mathrm{~A}$ adenosine receptor antagonists for the treatment of Parkinson's disease," Journal of Medicinal Chemistry, vol. 51, no. 22, pp. 7099-7110, 2008.

[9] R. Carbó-Dorca and L. Mercado, "Commentaries on quantum similarity (1): density gradient quantum similarity," Journal of Computational Chemistry, vol. 31, pp. 2195-2212, 2010.

[10] R. Carbó-Dorca, E. Besalú, and L. Mercado, "Communications on quantum similarity, part 3: a geometric-quantum similarity molecular superposition algorithm," Journal of Computational Chemistry, vol. 32, pp. 582-599, 2011.

[11] R. Carbó-Dorca and X. Gironés, "Foundation of quantum similarity measures and their relationship to QSPR: density function structure, approximations, and application examples," International Journal of Quantum Chemistry, vol. 101, no. 8, p. 20, 2005.

[12] R. Carbó-Dorca and X. Gironés, "Foundation of quantum similarity measures and their relationship to QSPR: density function structure, approximations, and application examples," International Journal of Quantum Chemistry, vol. 101, no. 1, pp. 8-20, 2005.

[13] L. Amatand and R. Carbó-Dorca, "Use of promolecular ASA density functions as a general algorithm to obtain starting MO in SCF calculations," International Journal of Quantum Chemistry, vol. 87, pp. 59-67, 2002.

[14] R. Carbó-Dorca and E. Besalú, "Communications on quantum similarity (2): a geometric discussion on holographic electron density theorem and confined quantum similarity measures," Journal of Computational Chemistry, vol. 31, pp. 2452-2462, 2010.

[15] R. Carbó-Dorca, M. Arnau, and L. Leyda, "How similar is a molecule to another? An electron density measure of similarity between two molecular structures," International Journal of Quantum Chemistry, vol. 17, pp. 1185-1189, 1980.

[16] P. Bultinck and R. J. Carbó-Dorca, "Molecular quantum similarity using conceptual DFT descriptors," Journal of Chemical Sciences, vol. 117, pp. 425-435, 2005.

[17] R. G. Parr and W. Yang, Density Functional Theory of Atoms and Molecules, Oxford University Press, New York, NY, USA, 1989.

[18] R. Vivas-Reyes, F. de Proft, P. Geerlings et al., "A DFT and HF quantum chemical study of the tin nanocluster [(RSn)12O14 $(\mathrm{OH}) 6] 2+$ and its interactions with anions and neutral nucleophiles: confrontation with experimental data," New Journal of Chemistry, vol. 26, no. 9, pp. 1108-1117, 2002.

[19] R. Mejia-Urueta, F. Nuñez-Zarur, and R. Vivas-Reyes, "Density functional study on electronic structures and reactivity in carbazol-oxadiazole dyads used in organic light emitting diodes," International Journal of Quantum Chemistry, vol. 112, pp. 2808-2815, 2012.

[20] F. de Proft, R. Vivas-Reyes, M. Biesemans, R. Willem, J. M. L. Martin, and P. Geerlings, "Density functional study of the complexation reaction of $\mathrm{Sn}\left(\mathrm{CH}_{3}\right)_{3} \mathrm{X}(\mathrm{X}=\mathrm{F}, \mathrm{Cl}, \mathrm{Br}$ and I) with Halide Anions," European Journal of Inorganic Chemistry, no. 20, pp. 3803-3810, 2003.

[21] A. Morales-Bayuelo and R. J. Vivas-Reyes, “Topological model to quantify the global reactivity indexes as local in DielsAlder reactions, using density function theory (DFT) and local 
quantum similarity (LQS)," Journal of Mathematical Chemistry, vol. 51, pp. 125-143, 2013.

[22] A. Morales-Bayuelo and A. J. Vivas-Reyes, "Theoretical model for the polarization molecular and Hückel treatment of PhosphoCyclopentadiene in an external electric field: Hirschfeld study," Journal of Mathematical Chemistry, vol. 51, pp. 1835-1852, 2013.

[23] A. Morales-Bayuelo and R. Vivas-Reyes, "Topological model on the inductive effect in alkyl halides using local quantum similarity and reactivity descriptors in the density functional theory," Journal Quantum Chemistry, vol. 2014, Article ID 850163, 12 pages, 2014.

[24] M. V. Putz, "Density functionals of chemical bonding," International Journal of Molecular Sciences, vol. 9, no. 6, pp. 1050-1095, 2008.

[25] M. V. Putz, "Markovian approach of the electron localization functions," International Journal of Quantum Chemistry, vol. 105, pp. 1-10, 2005.

[26] M. V. Putz, "Chemical action and chemical bonding," Journal of Molecular Structure: THEOCHEM, vol. 900, no. 1-3, pp. 64-70, 2009.

[27] E. Matito and M. V. Putz, "New link between conceptual density functional theory and electron delocalization," Journal of Physical Chemistry A, vol. 115, no. 45, pp. 12459-12462, 2011.

[28] G. Haskó, J. Linden, B. Cronstein, and P. Pacher, "Adenosine receptor signaling in the brain immune system," Nature Reviews Drug Discovery, vol. 7, pp. 759-770, 2008.

[29] A. Morales-Bayuelo, H. Ayazo, and R. Vivas-Reyes, "Threedimensional quantitative structure-activity relationship CoMSIA/CoMFA and LeapFrog studies on novel series of bicyclo [4.1.0] heptanes derivatives as melanin-concentrating hormone receptor R1 antagonists," European Journal of Medicinal Chemistry, vol. 45, no. 10, pp. 4509-4522, 2010.

[30] M. Ahumedo, A. Díaz, and R. Vivas-Reyes, “Theoretical and structural analysis of the active site of the transcriptional regulators LasR and TraR, using molecular docking methodology for identifying potential analogues of acyl homoserine lactones (AHLs) with anti-quorum sensing activity," European Journal of Medicinal Chemistry, vol. 45, pp. 608-615, 2010.

[31] X. Gironés and R. Carbó-Dorca, “TGSA-Flex: extending the capabilities of the Topo-Geometrical superposition algorithm to handle flexible molecules," Journal of Computational Chemistry, vol. 25, pp. 153-159, 2003.

[32] L. Chen, "Substructure and maximal common substructure searching," in Computational Medicinal Chemistry for Drug Discovery, P. Bultinck, H. de Winter, W. Langenaeker, and J. P. Tollenaere, Eds., p. 483, Marcel Dekker, New York, NY, USA, 2003.

[33] M. J. Frisch, G. W. Trucks, H. B. Schlegel et al., GAUSSIAN 09, Revision A.02, Gaussian, Wallingford, UK, 2009.

[34] A. D. Becke, "Density-functional exchange-energy approximation with correct asymptotic behavior," Physical Review A, vol. 38, p. 3098, 1988.

[35] C. Lee, W. Yang, and R. G. Parr, "Development of the ColleSalvetti correlation-energy formula into a functional of the electron density," Physical Review B, vol. 37, no. 2, pp. 785-789, 1988.

[36] W. J. Hehre, L. Radom, P. V. R. Schleyer, and J. A. Pople, Ab Initio Molecular Orbital Theory, John Wiley \& Sons, New York, NY, USA, 1986.
[37] R. Bultinck, X. Girones, and R. Carbó-Dorca, "Molecular quantum similarity: theory and applications," Reviews in Computational Chemistry, vol. 21, pp. 127-207, 2005.

[38] E. E. Hodgkin and W. G. Richards, "Molecular Similarity," Chemische Berichte, vol. 24, p. 1141, 1988.

[39] F. L. Hirshfeld, "Bonded-atom fragments for describing molecular charge densities," heoretica Chimica Acta, vol. 44, pp. 129138, 1977.

[40] F. de Proft, C. van Alsenoy, A. Peeters, W. Langenaeker, and P. Geerlings, "Atomic charges, dipole moments, and Fukui functions using the Hirshfeld partitioning of the electron density," Journal of Computational Chemistry, vol. 23, no. 12, pp. 1198-1209, 2002.

[41] M. Randic, "Design of molecules with desired properties," in Concepts and Applications of Molecular Similarity, M. A. Johnson and G. M. Maggiora, Eds., pp. 77-145, Wiley-Interscience, New York, NY, USA, 1990.

[42] G. Boon, C. van Alsenoy, F. de Proft, P. Bultinck, and P. Geerlings, "Molecular quantum similarity of enantiomers of amino acids: a case study," Journal of Molecular Structure: THEOCHEM, vol. 727, no. 1-3, pp. 49-56, 2005.

[43] P. G. Mezey, "Molecular physics: an international journal at the interface between chemistry and physics," Molecular Physics, vol. 96, pp. 169-178, 1999.

[44] L. Amat and R. Carbó-Dorca, "Quantum similarity measures under atomic shell approximation: first order density fitting using elementary Jacobi rotations," Journal of Computational Chemistry, vol. 18, pp. 2023-2039, 1997.

[45] R. G. Parr and W. T. Yang, "Density functional approach to the frontier-electron theory of chemical reactivity," Journal of the American Chemical Society, vol. 106, pp. 4049-4050, 1984.

[46] M. Putz, "Systematic formulations for electronegativity and hardness and their atomic scales within density functional softness theory," International Journal of Quantum Chemistry, vol. 106, pp. 361-389, 2006.

[47] P. Bultinck and R. Carbó-Dorca, "A mathematical discussion on density and shape functions, vector semispaces and related questions," Journal of Mathematical Chemistry, vol. 36, pp. 191200, 2004.

[48] R. M. Dreizler, Gross EKU Density Functional Theory, Springer, Berlin, Germany, 1990.

[49] R. F. Nalewajski and R. G. Parr, "Legendre transforms and Maxwell relations in density functional theory," The Journal of Chemical Physics, vol. 77, no. 1, pp. 399-407, 1982.

[50] M. Randic and C. L. Wilkins, "Graph theoretical approach to recognition of structural similarity in molecules," Journal of Chemical Information and Modeling, vol. 19, pp. 31-37, 1979.

[51] R. G. Parr and R. G. Pearson, "Absolute hardness: companion parameter to absolute electronegativity," Journal of the American Chemical Society, vol. 105, no. 26, pp. 7512-7516, 1983.

[52] M. Berkowitz and R. G. Parr, "Molecular hardness and softness, local hardness and softness, hardness and softness kernels, and relations among these quantities," The Journal of Chemical Physics, vol. 88, no. 4, pp. 2554-2557, 1988.

[53] R. G. Parr and L. J. Bartolotti, "Some remarks on the density functional theory of few-electron systems," Journal of Physical Chemistry, vol. 87, no. 15, pp. 2810-2815, 1983.

[54] A. C. Good and W. G. Richards, "Rapid evaluation of shape similarity using Gaussian functions," Journal of Chemical Information and Modeling, vol. 33, pp. 112-116, 1993. 
[55] M. Putz, "Semiclassical electronegativity and chemical hardness," Journal of Theoretical and Computational Chemistry, vol. 6, p. 33, 2007.

[56] R. F. W. Bader, Atoms in Molecules. A Quantum Theory, Oxford Science Publications/Clarendon Press, London, UK, 1990.

[57] P. Bultinck, S. Fias, C. van Alsenoy, P. W. Ayers, and R. CarbóDorca, "Critical thoughts on computing atom condensed Fukui functions," Journal of Chemical Physics, vol. 127, no. 3, Article ID 034102, 2007.

[58] K. Fukui, "Role of frontier orbitals in chemical reactions," Science, vol. 217, pp. 747-754, 1982.

[59] W. Yang, R. G. Parr, and R. Pucci, "Electron density, Kohn-Sham frontier orbitals, and Fukui functions," The Journal of Chemical Physics, vol. 81, no. 6, pp. 2862-2863, 1984. 

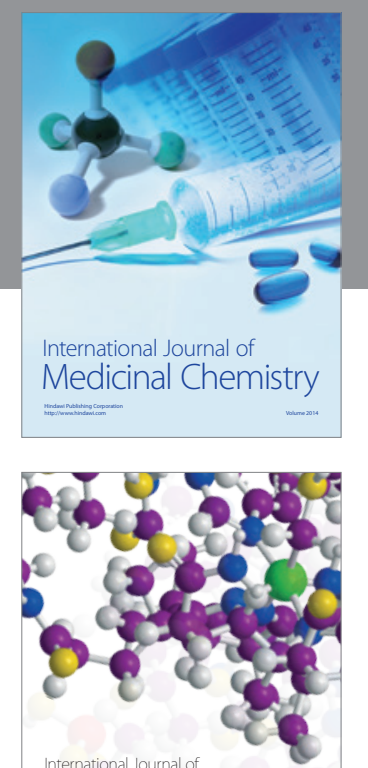

\section{Carbohydrate} Chemistry

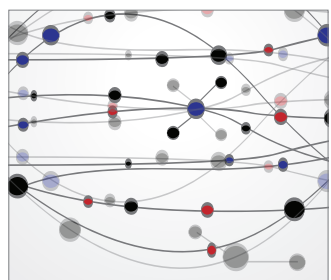

The Scientific World Journal
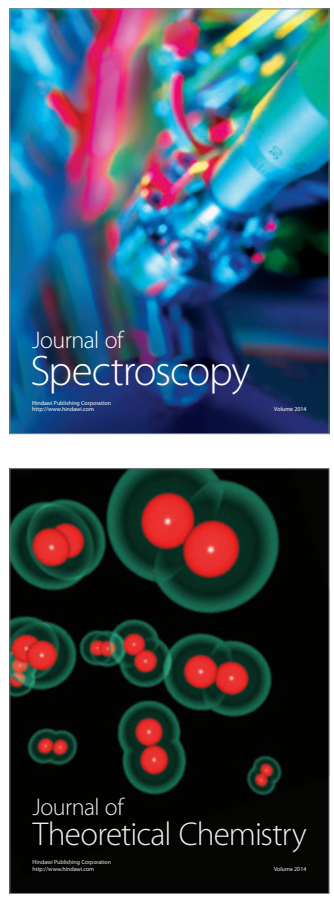
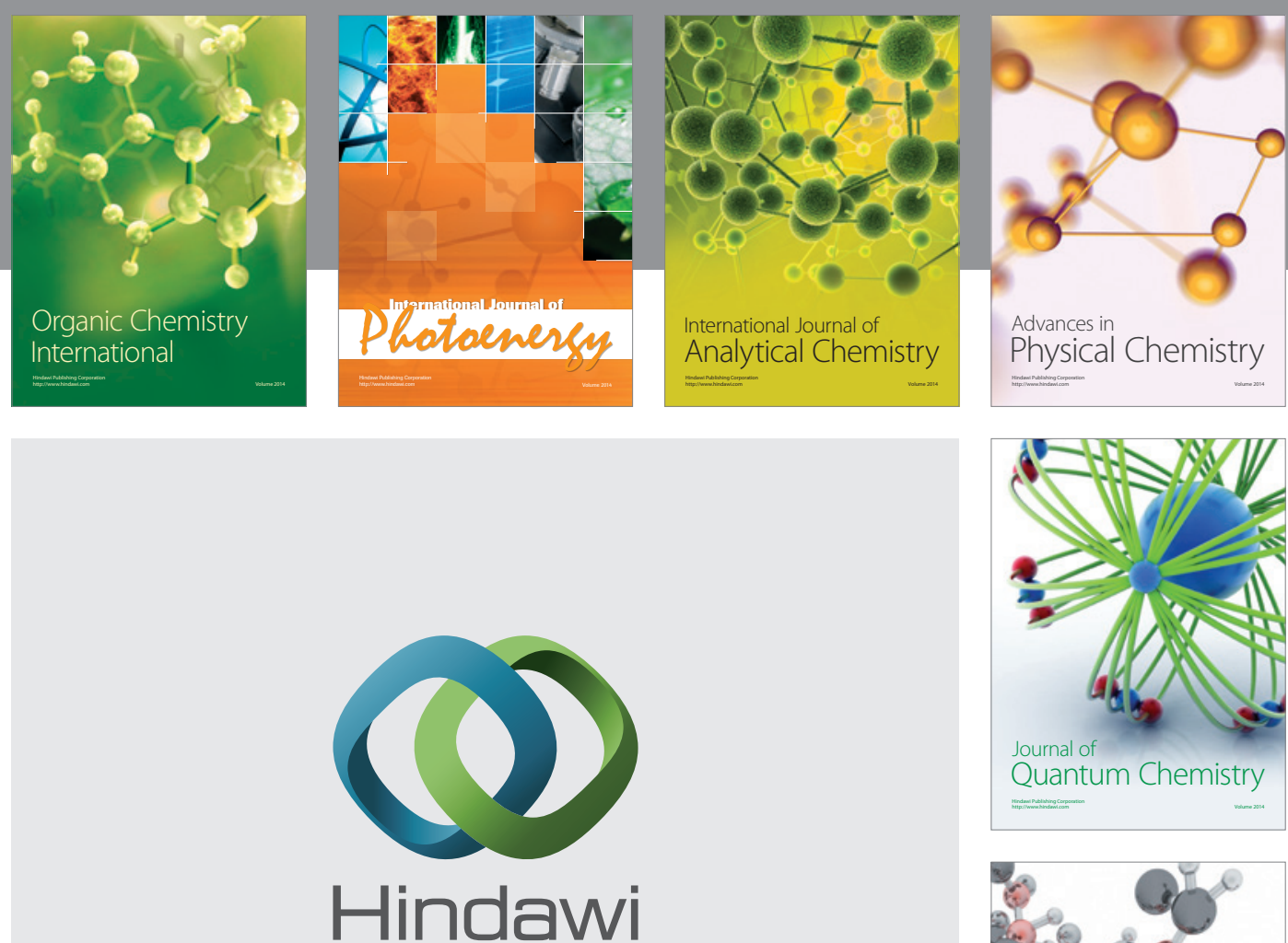

Submit your manuscripts at

http://www.hindawi.com

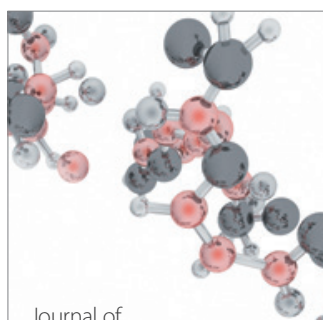

Analytical Methods

in Chemistry

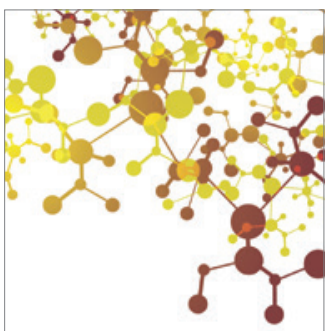

Journal of

Applied Chemistry

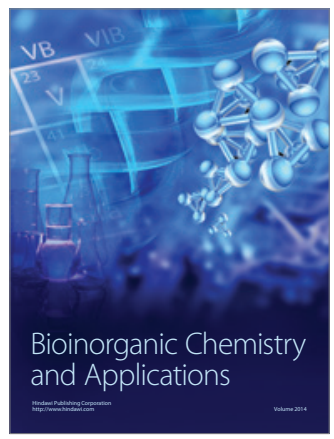

Inorganic Chemistry
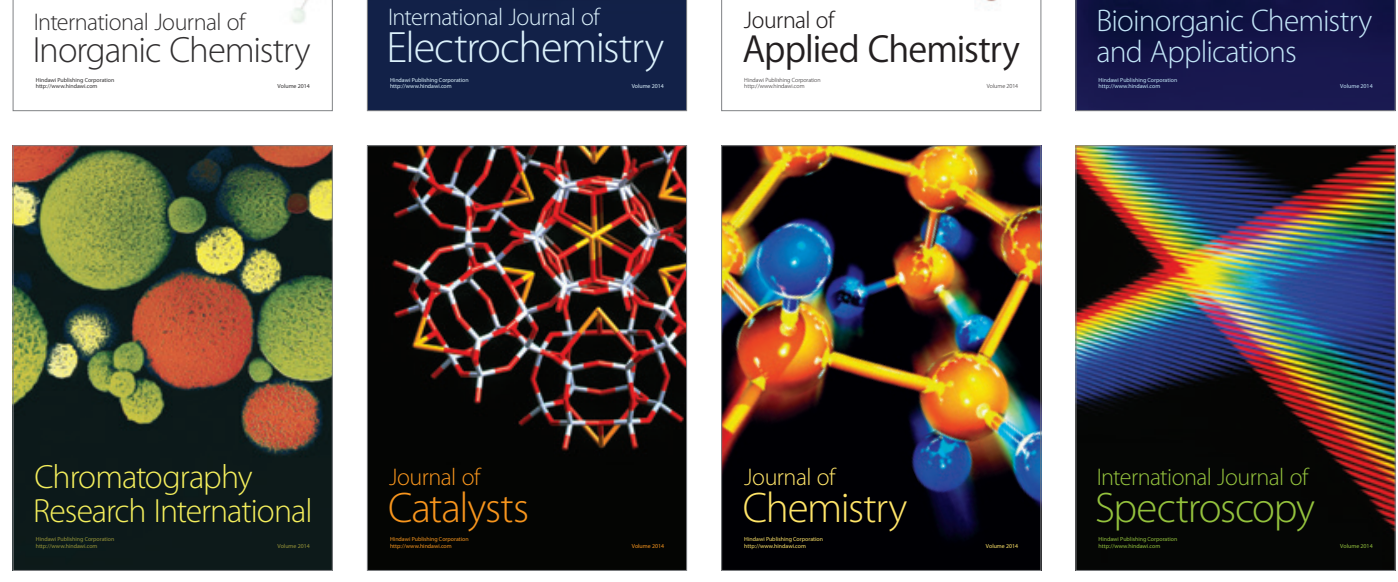